\title{
Establishment Background and Factors Affecting the Success of Telemedicine Provision
}

\author{
Ali Maher, ", Raziyeh Malmir ${ }^{1}$, Khalil Alimohamadzadeh ${ }^{1}$ \\ ${ }^{1}$ Department of Health Services Management, Tehran North Branch, Islamic Azad University, Tehran, IR Iran \\ *Corresponding Author: Ali Maher, Assistant Professor of Health Services Management, Department of Health Services Management, \\ Tehran North Branch, Islamic Azad University, Tehran, IR Iran. Tel: +98-9121979704, Email: dralimaher@gmail.com
}

\begin{abstract}
Introduction: Today, access to healthcare is considered a major indicator of community health. Telemedicine technology is an opportunity to increase access and improve the quality of healthcare in a country's development of a healthcare system. The establishment of a telemedicine system and the provision of healthcare in this way require a proper infrastructure, and multiple factors must be considered. This study investigated the areas of establishing telemedicine and the factors affecting its success in the Health Department of Iran's oil industry.

Methods: This study is objective and analytical (non-experimental) and used the research-applied method to collect information. The statistic community included all professionals and personnel of the Health Department of Iran's oil industry in 2014-2015. The establishment background and success factors of telemedicine provision were explored based on the study of 341 health experts and professionals. Data was analyzed by SPSS $_{16}$ and Lisral Software.

Results: The results show that both variables, i.e. establishment background (0.63) and organizational effectiveness factors (0.52), had a significant positive effect on the successful implementation of a telemedicine provision system. Furthermore, the factors of successful establishment of a telemedicine system (0.67) had a significant effect. Among the establishment background factors, financial structure (0.87) had the most significant effect on the successful establishment of a telemedicine system. Among the factors influencing success, organizational regulations and rules (0.83) were the most significant.

Conclusion: Based on the results of the current study, it is clear that giving the same attention to the establishment background and organizational effectiveness factors will lead to the successful implementation of telemedicine. It is recommended that the managers and policy makers of the country's health sector use and implement telemedicine in other areas as well. With proper planning, this could lead to increased community access to health services, savings in human and financial resources in the healthcare system, and reduced healthcare costs.
\end{abstract}

Keywords: Health Care, Treatment Services, Telemedicine

Article History: Received: 24 Nov. 2015; Accepted: 18 Jan. 2016; Online Published: 10 Apr. 2016

Cite this article as: Maher A, Malmir R, Alimohamadzadeh Kh. Establishment background and factors affecting the success of telemedicine provision. Int J Travel Med Glob Health. 2016;4(1):25-30.

\section{Introduction}

Today, access to healthcare is considered a major indicator of the health of a community [1]. Telemedicine technology is mentioned as a further opportunity to increase access and improve the quality of healthcare in a country's development of a healthcare system. Telemedicine will facilitate access to information for patients and medical professionals utilizing information and communications technology. Its goal is to increase quality and reduce the cost of healthcare through the online exchange of medical services. Telemedicine, in effect, is the use of electronic communications and telecommunications technology for service support and implementation such as remote clinical care, education, and training to professionals and patients in health-related fields, development of public health, and health management [2]. Telemedicine communication between hospitals and other medical institutions could lead to an overall improvement of healthcare services by focusing and aligning resources (professionals, hardware and software packets) [3].

The rise and expansion of Internet have created many changes in both science and industry; medical science and the healthcare industry are no exceptions to this rule. Internet has played a significant role in the development of medical services by making it possible to allow new and emerging technologies in the medical field $[4,5]$. Internet provides the possibility of taking advantage of doctors' knowledge and expertise in different geographical locations through the creation of a web-based information network. Through this, the concept of telemedicine was born, and it has made inevitable the creation of telemedicine services due to technical and geographical limitations to health service providers. E-Health and telemedicine provision are fields of science and technology in the healthcare industry that have increasing growth throughout the world $[6,7]$.

The geographic expansion of medical services and the need for specialized modern equipment to provide high quality service have encouraged medical care providers to take advantage of all the capabilities of medical technology and professionals to provide services in geographically remote areas. Telemedicine technology is able to provide facilities for the provision of healthcare services to geographically remote areas with standard quality using communication technology. In fact, this technology gives patients rapid access to better services, lower costs, and treatment courses, and it increases the overall quality of health services taking advantage of a database for the treatment history of patients.

Developing countries face a severe shortage of specialists in healthcare. There are few skilled medical professionals who use advanced medical technology such as scanners and other advanced diagnostic equipment. Generally, professionals work in university hospitals in large cities. The lack of medical specialists and limited opportunities for doctors in small, remote regional hospitals to consult with their colleagues in reference hospitals has led to a large number of unnecessary referrals [8-10].

Limited resources and few experts in various fields of medicine in addition to a lack of proper distribution of human resources have increased the need for telemedicine to be available in facilities in all parts of the country. Telemedicine has appeared in a variety of fields to help clinicians and 
healthcare managers to enable the same geographical capacities in providing healthcare services [11]. Telemedicine service has been attributed with $63 \%$ of the improvement possibility of treatment quality and $77 \%$ of the access to healthcare regardless of geographical conditions [12].

Iran's health service organizations, like those of other countries, need to establish a telemedicine technology infrastructure. Iran's oil industry is a developing industry in which it is essential that telemedicine services are applied with the aim of promoting the physical and mental health of employees and their families along with the geographical expansion of the industry. Given the geographical expansion of oil industry operations centers and due to events that may be created in oil-rich areas for employees, healthcare can be provided to affected people in the region in the shortest time through the launch of a Telemedicine Center. This project allows researchers access to two important goals: reducing the volume of patients referring to medical centers, and reducing absenteeism in order to perform diagnostic operations.

The adoption of telemedicine technology as a strategy of structural transformation in healthcare faces many challenges [13]. The present study investigated factors affecting the success of the implementation of telemedicine technology in the Health Department of Iran's oil industry as the only healthcare organization in Iran that makes use of this technology while investigating the background of telemedicine establishment. Accordingly, three interrogative fields resulting from pondering establishment infrastructures and effective factors on the success of telemedicine provision were considered:

A) Establishment infrastructures that include 1) financial infrastructures, 2) technological infrastructures, 3) organizational infrastructures, and 4) telemedicine human resource management in the Health Department of Iran's oil industry.

B) Factors that affect success and explain 1) organizational rules and regulations, 2) budget allocation, 3) acculturation, 4) tools and equipment, 5) training of manpower, 6) telemedicine media representation in the Health Department of Iran's oil industry.

C) Is there a relationship between the establishment infrastructures and the factors affecting telemedicine success in the Health Department of Iran's oil industry?

This study was conducted in response to the abovementioned issues and assessed the factors affecting the success of telemedicine in the Health Department of Iran's oil industry. The results can be useful in identifying the strengths and weaknesses of the program and providing technical and managerial solutions in all relevant areas in other country's healthcare and medical organizations.

\section{Methods}

The objective and analytic (non-experimental) study is research-applied. The study's statistic community included all professionals and personnel of the Health Department of Iran's oil industry in 2014-2015.

Multistage random-cluster sampling method was used in this study. The areas were chosen randomly and then sampling was carried out in each area. Examples of this design were determined based on the list of managers and staff of the Health Department of Iran's oil industry (and using Morgan's Table) whose number was estimated to be 341 subjects.

The research instrument was a researcher-made questionnaire for collecting information. The content validity of the questionnaire was checked through surveying the success of the establishment background and the factors affecting success in providing telemedicine to the Health Department of Iran's oil industry by obtaining feedback from professors and experts. Its reliability was checked by performing a pre-test step and measuring Cronbach's alpha coefficient, which equaled 0.974. Thus, the measuring devices have excellent reliability. The construct validity and reliability of the main variables were assessed using factor analysis; the results indicated the construct and composite reliability of the variables (Table 1). The questionnaire included two groups of general and specific questions. General questions asked the age, gender, and education level of the participants.

Table 1. Summary of constructs and their measurement properties

\begin{tabular}{|c|c|c|c|}
\hline Construct & Construct Reliability $^{\text {a }}$ & $\mathbf{A V E}^{\mathbf{b}}$ & Cronbach's Alpha \\
\hline Financial infrastructure $(\mathrm{FI})$ & 0.87 & 0.62 & 0.78 \\
\hline Technological infrastructure (TI) & 0.85 & 0.65 & 0.80 \\
\hline Organizational infrastructure (OI) & 0.89 & 0.71 & 0.83 \\
\hline Human resources Infrastructure (HRI) & 0.78 & 0.69 & 0.79 \\
\hline Rules and regulations (RR) & 0.77 & 0.67 & 0.75 \\
\hline Budget formulation (BF) & 0.80 & 0.72 & 0.76 \\
\hline Acculturation (AC) & 0.84 & 0.60 & 0.80 \\
\hline Equipment (EQ) & 0.86 & 0.73 & 0.82 \\
\hline Human resource training (HRT) & 0.78 & 0.70 & 0.79 \\
\hline Media representations (MR) & 0.90 & 0.75 & 0.85 \\
\hline
\end{tabular}

The second part of the questionnaire included 102 specific questions related to the variables based on the objectives and research questions. Of these, 44 questions were related to the evaluation criteria of establishment infrastructures and comprised the financial infrastructure measurement (7 questions), technological infrastructures (13 questions), organizational infrastructures (12 questions), and human resource management infrastructures (11 questions) and 58 questions related to the measurement of success factors comprising the measurement of organizationalgovernmental laws and regulations development (7 questions), budget (9 questions), acculturation (12 questions), equipment and tools (9 questions), manpower training (11 questions), and media representation (10 questions).

Questions were scored on a 5-point Likert scale that was designed and used given the nominal-ranking scale based on responses (very low, low, medium, high, or very high; 
grading for each option was $1,2,3,4$, or 5, respectively. With this valuation method that is intended for very low options (value or quantity 1) and for very high options (value or quantity 5), the grading or ranking scale converts into a quasi-space scale which makes it possible for researchers to use parametric and structural equations modeling.

This study used the SPSS version 16 and LISREL software, Kolmogorov-Smirnov test, and Pearson correlation to analyze data collected by questionnaires, the structural equation modeling test to analyze the confirmatory factor, and path analysis to test the research hypotheses.

\section{Results}

In this descriptive study, $64.35 \%$ of respondents were female and $35.65 \%$ male, according to data from 341 questionnaires completed by professionals and personnel of the Health Department of Iran's oil industry; $52.46 \%$ of respondents had a bachelor's degree, $23.48 \%$ had a $\mathrm{PhD}$, $12.7 \%$ had an associate's degree, $4.06 \%$ had a master's degree, $4.06 \%$ had a high school diploma, and $3.77 \%$ had a professional $\mathrm{PhD}$ degree. Some $15.94 \%$ of respondents were between the ages of 25 and 30 years, $27.83 \%$ were between 31 and 36 years, $23.77 \%$ were between 37 and 42 years, $24.35 \%$ were between 43 to 48 years, and $8.12 \%$ were between 49 and 54 years of age.
The correlation coefficients matrix results between the independent variables of establishment and the success factors showed that of 24 coefficients examined, 12 were significant at a confidence level of $99 \%, 5$ coefficients were significant at a $95 \%$ confidence level, and 7 were not significant at a 95\% confidence level. The maximum correlation coefficient was significant between the two variables human resources infrastructure and budget formulation (0.797), which is indicative of a strong positive and significant relationship between these variables. The minimum correlation coefficient was significant between the two variables technological infrastructure and budget formulation (0.114), which represents a poor positive and significant relationship between them (Table 2).

The confirmatory factor analysis test was used to analyze the internal structure of the questionnaire and to discover the constituent elements of each variable. As shown in Table 3, the fitting indicators obtained from this analysis in all research variables showed a good fitness. Results of the confirmatory factor analyses of the variables are listed in Table 3. As can be seen, all factorial loads in all variables were outside the limits of +2 and -2 . Thus, according to the results, all factorial loadings are significant at $99 \%$. As a result, all indicators defined for variables were significant and considered as indicators.

Table 2. The correlation matrix between research variables (sample size $=341$ )

\begin{tabular}{|c|c|c|c|c|}
\hline $\begin{array}{l}\text { Establishment Factors (EF) } \\
\text { Success Factors (SF) }\end{array}$ & $\begin{array}{c}\text { Financial } \\
\text { Infrastructure }\end{array}$ & $\begin{array}{l}\text { Technological } \\
\text { Infrastructure } \\
\end{array}$ & $\begin{array}{l}\text { Organizational } \\
\text { Infrastructure }\end{array}$ & $\begin{array}{l}\text { Human Resource } \\
\text { Infrastructure }\end{array}$ \\
\hline Laws and regulations & $0.124^{*}$ & $0.132^{*}$ & $0.200^{* *}$ & $0.731^{* *}$ \\
\hline Budget formulation & $0.124^{*}$ & $0.114^{*}$ & $0.251^{* *}$ & $0.797^{* *}$ \\
\hline Acculturation & 0.091 & $0.115^{*}$ & $0.210^{* *}$ & $0.775^{* *}$ \\
\hline Tools, equipment & 0.103 & $0.147^{* *}$ & $0.253^{* *}$ & $0.773^{* *}$ \\
\hline Manpower training & 0.102 & 0.105 & $0.200^{* *}$ & $0.794^{* *}$ \\
\hline Media representation & 0.105 & 0.031 & 0.024 & $0.729^{* *}$ \\
\hline
\end{tabular}

${ }^{*}$ Significance at 0.05 level. ${ }^{* *}$ Significance at 0.01 level.

Table 3. Fitting indicators of research variables

\begin{tabular}{|c|c|c|c|c|c|c|}
\hline $\begin{array}{ll}\text { Variables } & \text { Fitness Indicators } \\
\end{array}$ & $X^{2} / \mathbf{d f}$ & GFI & AGFI & CFI & NFI & RMSEA \\
\hline Financial infrastructure(FI) & 1.93 & 0.98 & 0.96 & 0.98 & 0.96 & 0.052 \\
\hline Technological infrastructure (TI) & 2.24 & 0.95 & 0.91 & 0.95 & 0.92 & 0.060 \\
\hline Organizational infrastructure (OI) & 2.23 & 0.95 & 0.90 & 0.98 & 0.97 & 0.051 \\
\hline Human resources infrastructure (HRI) & 1.56 & 0.98 & 0.95 & 1.00 & 0.99 & 0.041 \\
\hline Rules and regulations(RR) & 2.25 & 0.99 & 0.95 & 1.00 & 0.99 & 0.060 \\
\hline Acculturation (AC) & 2.47 & 0.95 & 0.91 & 0.98 & 0.97 & 0.056 \\
\hline Equipment (EQ) & 1.73 & 0.99 & 0.95 & 1.00 & 1.00 & 0.046 \\
\hline Human resources training (HRT) & 2.41 & 0.98 & 0.91 & 1.00 & 0.99 & 0.079 \\
\hline Media representations (MR) & 2.89 & 0.95 & 0.9 & 0.99 & 0.98 & 0.037 \\
\hline
\end{tabular}

Table 4. Results of factor analyses of research variables

\begin{tabular}{|c|c|c|c|}
\hline Variables & Indicator with most Important role in Defining and Measuring Variables & $\begin{array}{c}\text { Factor } \\
\text { Loading }\end{array}$ & $\begin{array}{c}\text { Significance } \\
\text { Coefficient }\end{array}$ \\
\hline Financial infrastructure (FI) & Indicator of approving budget and financial management & 0.73 & 12.95 and 12.97 \\
\hline Technological infrastructure (TI) & Indicator of availability of the medical data transmission network for diagnosis & 0.73 & 14.29 \\
\hline Organizational infrastructure (OI) & Indicator of organizational need and motivation & 0.96 & 24.02 \\
\hline Human resources infrastructure (HRI) & Indicator of methods implementation and leadership policies & 0.95 & 23.076 \\
\hline Rules and regulations (RR) & Indicator of focusing on communications law and compliance of cyberspace legal provisions & 0.96 & 24.12 \\
\hline Acculturation (AC) & Institutionalizing the education pattern of network concept & 0.87 & 19.75 \\
\hline Equipment (EQ) & Satellite telecommunications equipment & 0.97 & 26.15 \\
\hline Human resource training (HRT) & Training protocols formulation & 0.95 & 23.43 \\
\hline Media representations (MR) & True reflection of media & 0.92 & 22.42 \\
\hline
\end{tabular}




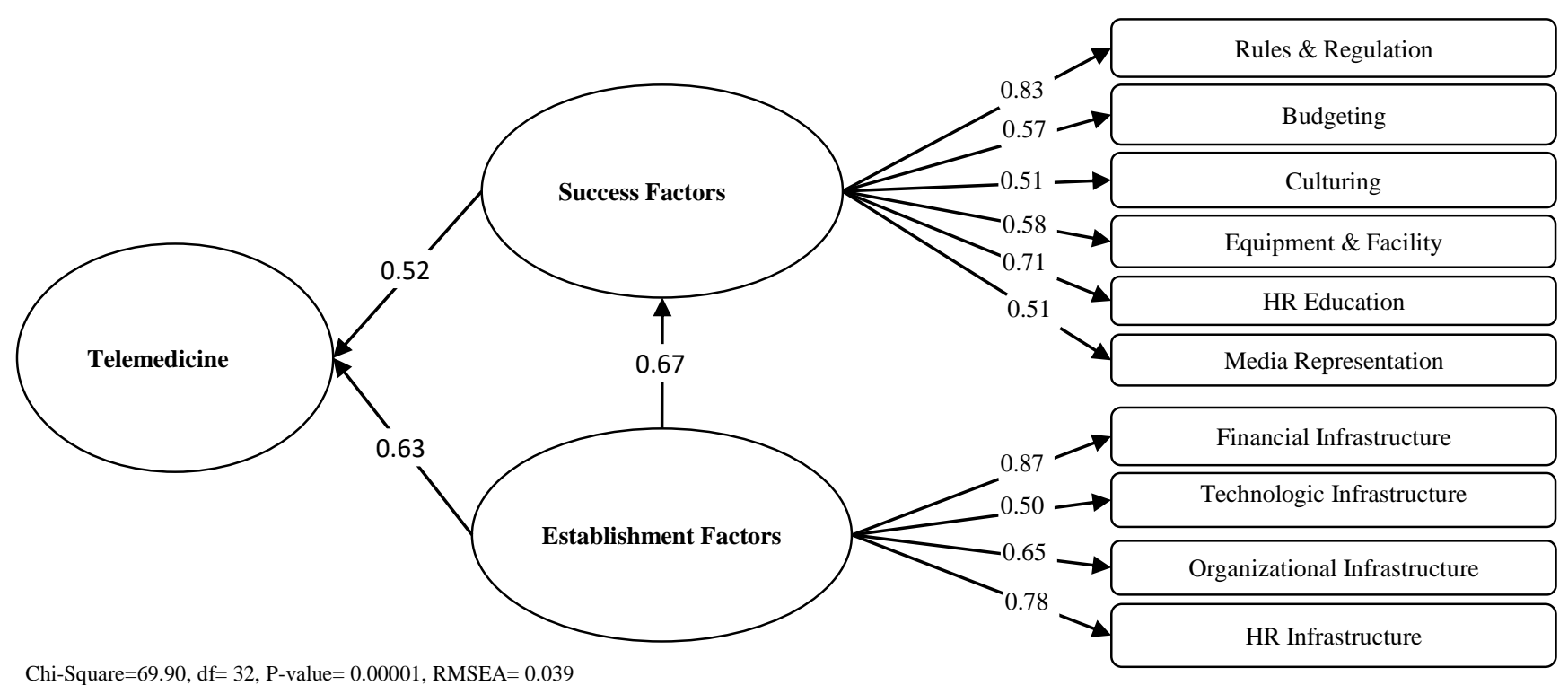

Figure 1. Fitness of the conceptual model - Structural part (standard)

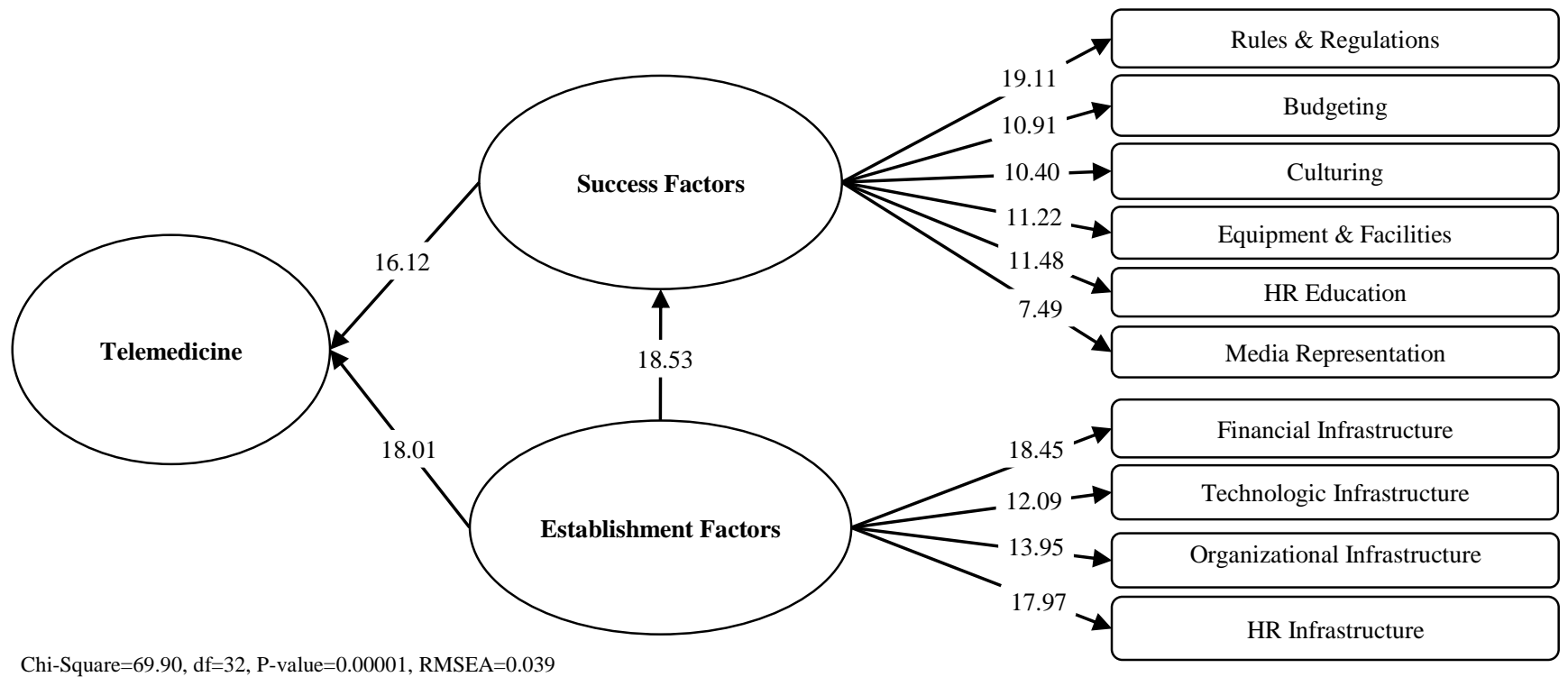

Figure 2. Fitness of the conceptual model-structural part (significance)

The following table shows the indicators with the most important roles in defining and measuring the variables.

With the aim of detecting whether theoretical relationships between variables can be approved (in the conceptual framework formulation intended by the researcher) via data, the structural part of the conceptual model of relationships between the inner and outer variables need to be evaluated. The structural part of the fitness of the conceptual model is shown in Figure 1 and Figure 2.

Since the fitness indicators obtained from the conceptual model (chi-square to degree of freedom 2.18, GFI $=0.98$, $\mathrm{AGFI}=0.97, \mathrm{CFI}=1.00, \mathrm{NFI}=0.99$, and $\mathrm{RMSEA}=0.039$ ) are higher than the limit, the conceptual model has good fitness; in other words, the data collected explains and demonstrates well the relationships of the conceptual model. Table 4 shows the summary results from the structural equation modeling in which all obtained factor loadings (path coefficients) are significant at a confidence level of
$99 \%$ with respect to the results.

Based on the results listed in Table 5, this study showed that both variables of establishment background with a factor loading of 0.63 and organizational effectiveness factors with a factor loading of 0.52 at a confidence level of $99 \%$ have a significant positive effect on the successful implementation of the telemedicine system. Also, all variables in both sections have a positive and meaningful impact on the successful establishment of a telemedicine system at a confidence level of $99 \%$. Of the background of establishment, financial structure $(0.87)$ had the most positive and significant impact on the successful establishment of a telemedicine system, and of the factors affecting success, organizational rules $(0.83)$ was the most significant factor. The resulting hypothesis of this study also indicates that the successful establishment factors for the telemedicine system have a significant positive impact (0.67) on the factors influencing its successful establishment. 
Table 5. Path coefficients and t-statistics

\begin{tabular}{|c|c|c|c|c|}
\hline Hypotheses & Independent Variable Dependent Variable & Path Coefficient $(\beta)$ & t-Statistic & Result of Hypothesis \\
\hline Main hypothesis 1 & Establishment background Successful implementation & & & Confirmed \\
\hline Main hypothesis 2 & Factors affecting successful implementation & & & Confirmed \\
\hline Sub- hypothesis 1 & Human resources infrastructure Successful establishment & & & Confirmed \\
\hline Sub-hypothesis 2 & Financial structure Successful establishment & & & Confirmed \\
\hline Sub-hypothesis 3 & Technological structure Successful establishment & & & Confirmed \\
\hline Sub-hypothesis 4 & Organizational structure Successful establishment & & & Confirmed \\
\hline Sub-hypothesis 5 & Laws and regulations Successful establishment & & & Confirmed \\
\hline Sub-hypothesis 6 & Budget Successful establishment & & & Confirmed \\
\hline Sub-hypothesis 7 & Acculturation Successful establishment & & & Confirmed \\
\hline Sub-hypothesis 8 & Equipment Successful establishment & & & Confirmed \\
\hline Sub-hypothesis 9 & Manpower training Successful establishment & & & Confirmed \\
\hline Sub-hypothesis 10 & Media representation of successful establishment & & & Confirmed \\
\hline Hypotheses derived from the study & Factors affecting successful establishment & & & Confirmed \\
\hline
\end{tabular}

${ }^{*}$ Significance at 0.05 level ${ }^{* *}$ Significance at 0.01 level

\section{Discussion}

The results showed that both variables, i.e. establishment background and organizational effectiveness factors at a confidence level of $99 \%$ have a significant positive effect on the successful implementation of the telemedicine system. The study also revealed the important role of reviewing the establishment background and factors affecting the success of telemedicine provision. In previous studies conducted inside the country and abroad, researchers analyzed the different factors affecting the provision of telemedicine as well as its benefits and impact.

The results of this study suggest that the financial structure (with a factorial loading of 0.83 ) has the greatest impact on the successful establishment of a telemedicine system. In 2012, Rahimzadeh showed that $16.98 \%$ of experts believe the initial costs to be the biggest barrier to the use of telemedicine [14]. In effect, economic considerations are the main challenges to implementing such projects. In most cases, the problem for project sustainability is the initial investment and overhead that normally should be borne by the service provider. However, the economic savings resulting from the use of telemedicine are more beneficial to the clients. This financial cycle should be completed for the sustainability of a telemedicine project, and insurance companies can play a good role in this regard. The discussed economic cycle is completed in the Health Department of Iran Oil Company; this organization is both a service provider and a client, so it would be economically justified to launch telemedicine clinics for this company.

Undoubtedly, technical and technological issues are a prerequisite for the installation of a telemedicine system. This study found that technological structure with the factorial loading of 0.50 has a significant positive effect on the successful establishment of a telemedicine system. Porazin in his study also recognized technology resources as one of the telecommunication lines, wireless networks, and the Internet as the most important sources for the consultation network establishment of a telemedicine system [15]. Kannan in his study suggested that the technical and communications equipment including telecommunication lines and wireless technology are among the most important resources for the establishment of a telemedicine consultation system [16]. Judi et al. noted in their study that, in addition to human resources, telecommunication lines and communications equipment are the most important technological factors of a telemedicine system [17]. The study also showed that the indicator of access to the medical data transmission network for diagnosis with a factorial loading of 0.73 plays the most important role in the technological structure; these results are consistent with those of Fayyazi et al. [18]. Therefore, considering the benefits of telemedicine, planning should be taken on by the managers of the Health Department of Iran's oil industry to supply equipment for the establishment of a telemedicine system.

One of the most important factors in the successful establishment of telemedicine is manpower training as reported by the majority of studies reviewed by the researcher. Kurd and Mirinejad in 2014 showed that the lack of knowledge of doctors and staff is a problem for the success of a telemedicine system [19]. The research of Dargahi and Razavi in 2005 also showed that with the successful operation and implementation of telemedicine technology in subsidiary hospitals, from a total of 82 faculty members, $94 \%$ believed that continuing education programs for physicians and employees were necessary [20]. According to the results of these studies, the training of human resources with a factorial loading of 0.71 has a positive and meaningful impact on the successful establishment of a telemedicine system. Thus, the oil industry's health managers, by planning the necessary training courses, can increase the ability of their own employees in this area.

The studies of Bangert, Doktor [21], Yellowlees [22] and the present study all showed that the structure of human resources has a positive and significant role in the success of a telemedicine system. Despite the fact that one of the management challenges in changing health service provision models or introducing a new model is the resistance of staff to changes. Support and active participation by senior managers can be a factor for success in the workforce structure. Goal setting is another managerial strategy for reducing resistance to change in centers where staff has been newly recruited have not adapted to the routines.

\section{Conclusion}

Telemedicine technology is an opportunity to increase access and improve the quality of healthcare in the country's development of a healthcare system. Healthcare systems can use their knowledge and services in these areas and use telemedicine to set up consultation sessions between physicians rather than requiring the physical presence of specialists in remote areas. Moreover, the need to establish new healthcare centers is reduced by the use of telemedicine in healthcare systems, and this saves much in the financial and human resource areas of a healthcare system. In addition, the establishment of a telemedicine system can increase the community's access to health services and reduce healthcare 
costs. Thus, based on the results of this study, it is suggested that other healthcare organizations also carry out the necessary measures to investigate the background of the establishment and success factors of the Iran Oil Company to establish a telemedicine system. In conclusion, the following suggestions are offered to improve the establishment and success in telemedicine provision:

1. Planning and efforts should be undertaken to increase public awareness and understanding of a telemedicine system.

2. Necessary skills training for doctors and health personnel in the use of a telemedicine system should be established.

3. Telemedicine should be included in the curriculum of medical students.

4. Policies, guidelines, and standards for the establishment of a telemedicine system in the country should be formulated.

5. Efforts should be made to reduce the legal restrictions on the provision of telemedicine.

6. Planning and efforts should be undertaken to provide the equipment required for a telemedicine system in the country.

7. Obstacles to the organizational structure in the country's different parts of healthcare should be removed with the aim of successfully establishing a telemedicine system.

\section{Acknowledgments}

This article is extracted from a Health Services Management Master's thesis.

\section{Authors' Contributions}

All authors contributed equally in the preparation of this paper.

\section{Financial Disclosure}

The authors declare they have no financial disclosures.

\section{Funding/Support}

This research has been supported by the Islamic Azad University, Tehran North Branch of Tehran.

\section{References}

1. Torani S, Khammarnia M, Delgoshaei B. The ability of specialized hospitals of iran university of medical sciences in establishing remote medical advice. Director General. 2012;8(6):785-94.

2. Chabat F, Hansell DM, Yang G-Z. Computerized decision support in medical imaging. IEEE Eng Med Biol Mag. 2000;19(5):89-96.
3. Cadilhac DA, Moloczij N, Denisenko S, Dewey H, Disler P, Winzar $\mathrm{B}$, et al. Establishment of an effective acute stroke telemedicine program for Australia: protocol for the Victorian Stroke Telemedicine project. Int J Stroke. 2014;9(2):252-8.

4. Cascardo D. Telemedicine: Advancing from Idea to Implementation. J Med Pract Manage. 2015;31(2):82-4.

5. Goodarzi M, Torabi M, Safdari R, Dargahi H, Naeimi S. Innovation Network Development Model in Telemedicine: A Change in Participation. Healthc Inform Res. 2015;21(4):265-70.

6. Pinciroli F, Corso M, Fuggetta A, Masseroli M, Bonacina S, Marceglia S. Telemedicine and e-health. IEEE Pulse. 2011;2(3):6270.

7. Dávalos ME, French MT, Burdick AE, Simmons SC. Economic evaluation of telemedicine: review of the literature and research guidelines for benefit-cost analysis. Telemed J E Health. 2009;15(10):933-48.

8. Bashshur RL, Reardon TG, Shannon GW. Telemedicine: a new health care delivery system. Annu Rev Public Health. 2000;21(1):613-37.

9. Bashshur RL. Telemedicine effects: cost, quality, and access. J Med Syst. 1995;19(2):81-91.

10. Ostbye T, Hurlen P. The electronic house call. Consequences of telemedicine consultations for physicians, patients, and society. Arch Fam Med. 1997;6(3):266-71.

11. Scott RE. e-Records in health--preserving our future. Int $\mathbf{J}$ Med Inform. 2007;76(5-6):427-31.

12. Hayavi Haghighi M, Alipour J, Mastaneh Z, Mouseli L. Feasibility study of telemedicine implementation in Hormozgan university of medical sciences. Bimonthly Journal of Hormozgan University of Medical Sciences. 2011;15(2):128-37.

13. Ramalingam K. Chief Telemedicine Technical Services in Amrita Institute of Medical sciences \& Research Center. 2002.

14. Rahimzadeh E, Rahimzade S, Azadi S, Amani F. Feasibility of application and establishment of telemedicine in Ardabil's Imam Khomeini Hospital. Student Res Commit Ardabil Med Sci Univ J. 2012;2(2):5-10. Persian.

15. Porazin SH. Estimated resources to implement telehealth in Iran Proceedings of the 4th Regional Conference on eHealth; 18-19 Sep. 2004 Tehran: Health and Medical Education Secretariat Takfab; 2004. p. 13. Persian.

16. Kannan S. Utilization of Telehealth in India 2008. Available from: https://mpra.ub.uni-uenchen.de/15001/1/MPRA_paper_15001.pdf.

17. Judi HM, Razak A, Sha'ari N, Mohamed H. Feasibility and critical success factors in implementing telemedicine. Inf Technol J. 2009;8(3):326-32.

18. Fayyazi A, Ghaedamini R, Azizian F, Tofighi Sh. Comparison of physicians and paramedics' conception on the use of telemedicine in Shahrekord's Ayatollah Kashani Hospital. In: Sciences TUoM, editor. The First International Symposium on Electronic Hospital and Telemedicine; Tehran: Tapko Congress Center; 2010. Persian.

19. Kurd B, Mirinejad E. Study of Effective Factors on Implementation of Telemedicine in Zahedan University of Medical Sciences. Asian J Res Soc Sci Humanit. 2014;4(8):287-302.

20. Dargahi H, Razavi SM. The role of organizational culture in the implementation of telemedicine technology in health care centers of Tehran University of Medical Sciences in 2003-2004. Tehran Univ Med Sci J. 2003;63(2):99-107. Persian.

21. Bangert D, Doktor R. The role of organization culture in management of clinical e-health systems, proceeding of 36th Hawaii international Conference on System Sciences. 2003 Jan 6-9: Hawaii. USA.

22. Yellowlees P. Successful development of telemedicine systemsseven core principles. J Telemed Telecare. 1997;3(4):215-22. 